\title{
Herpes Zoster Infection in Patients With Ulcerative Colitis Receiving Tofacitinib
}

\author{
Kevin L. Winthrop, MD, MPH, ${ }^{*}$ Gil Y. Melmed, MD, MS, ${ }^{+}$Séverine Vermeire, MD, $P h D,{ }^{\ddagger}$ Millie D. Long, $M P H_{,}{ }^{\S}$ \\ Gary Chan, PharmD, MS," Ronald D. Pedersen, MS," Nervin Lawendy, PharmD," Andrew J. Thorpe, PhD," \\ Chudy I. Nduaka, DVM, PhD," and Chinyu Su, MD"
}

\begin{abstract}
Background: Tofacitinib is an oral, small molecule Janus kinase inhibitor that is being investigated for ulcerative colitis (UC). Tofacitinib is approved for rheumatoid arthritis and psoriatic arthritis, where it has been shown to increase herpes zoster (HZ) risk. We evaluated HZ risk among UC patients using tofacitinib.
\end{abstract}

Methods: HZ cases were identified in tofacitinib phase II/III/ongoing, open-label, long-term extension (OLE) UC trials. We calculated HZ incidence rates (IRs) per 100 patient-years of tofacitinib exposure within phase III maintenance (Maintenance Cohort) and phase II/III/OLE (Overall Cohort) studies, stratified by baseline demographics and other factors. HZ risk factors were evaluated in the Overall Cohort using Cox proportional hazard models.

Results: Overall, $65(5.6 \%)$ patients developed HZ. Eleven patients had multidermatomal involvement ( 2 nonadjacent or 3-6 adjacent dermatomes), and 1 developed encephalitis (resolved upon standard treatment). Five (7.7\%) events led to treatment discontinuation. HZ IR (95\% confidence interval [CI]) in the Overall Cohort was 4.07 (3.14-5.19) over a mean (range) of 509.1 (1-1606) days, with no increased risk observed with increasing tofacitinib exposure. IRs (95\% CI) were highest in patients age $\geq 65$ years, 9.55 (4.77-17.08); Asian patients, 6.49 (3.55-10.89); patients with prior tumor necrosis factor inhibitor (TNFi) failure, 5.38 (3.86-7.29); and patients using tofacitinib 10 mg twice daily, 4.25 (3.18-5.56). Multivariate analysis identified older age and prior TNFi failure as independent risk factors.

Conclusions: In tofacitinib-treated UC patients, there was an elevated risk of HZ, although complicated HZ was infrequent. Increased HZ rates occurred in patients who were older, Asian, or had prior TNFi failure (NCT00787202, NCT01465763, NCT01458951, NCT01458574, NCT01470612).

Key Words: herpes zoster, shingles, tofacitinib, ulcerative colitis, Janus kinase, safety

\section{INTRODUCTION}

Herpes zoster (HZ) represents the reactivation of varicella zoster virus (VZV), and it becomes more common with increasing age and the waning of VZV-specific cell-mediated immunity. ${ }^{1}$ Approximately one-third of individuals in the United States will develop $\mathrm{HZ}$ within their lifetime. ${ }^{1-3}$ Although $\mathrm{HZ}$ is generally self-limited, it manifests with substantial morbidity for many patients, and in approximately $15 \%$ of patients it causes longterm disability in the form of postherpetic neuralgia. ${ }^{1}$ More rarely, disseminated $\mathrm{HZ}$ can occur within immunosuppressed patients,

Received for publications December 7, 2017; Editorial Decision February 25, 2018. From the *Oregon Health \& Science University, Portland, Oregon; ${ }^{\dagger}$ CedarsSinai Medical Center, Los Angeles, California; ${ }^{\star}$ Department of Gastroenterology, University Hospitals Leuven, Leuven, Belgium; ${ }^{\S}$ Center for Gastrointestinal Biology and Disease, University of North Carolina, Chapel Hill, North Carolina; 'Pfizer Inc., Collegeville, Pennsylvania

Conflicts of interest: K.W. has received consulting and investigator fees from Pfizer Inc. G.M. has received consulting fees from AbbVie, Boehringer Ingelheim, Celgene, Genentech, Janssen, Pfizer Inc., Samsung Bioepis, Takeda, and UCB; and has received support for research from Prometheus Labs. S.V. has received grant support from AbbVie, MSD, and Takeda; has received speaker fees from AbbVie, MSD, Takeda, Ferring, Dr Falk Pharma, Hospira, and Tillots; and has received consulting fees from AbbVie, MSD, Takeda, Ferring, Genentech/Roche, Shire, Pfizer Inc., Galapagos, Mundipharma, Hospira, Celgene, Second Genome, and Janssen. M.L. has received consulting fees from AbbVie, UCB, Pfizer, Takeda, and Target potentially leading to encephalitis and death. The increased risk of $\mathrm{HZ}$ in some autoimmune conditions is well described. In patients with rheumatoid arthritis (RA), the risk of $\mathrm{HZ}$ is elevated approximately 2 -fold ${ }^{4}$ compared with similarly aged individuals within the general population. Patients with inflammatory bowel disease (IBD) have also been shown to be at increased risk for $\mathrm{HZ}$, with recent population-based data suggesting an up to 2-fold risk elevation over the general population. ${ }^{5}$ Additional studies in IBD have shown increased risk with therapies such as corticosteroids, thiopurines, and tumor necrosis factor inhibitor (TNFi) therapy.,

Pharmasolutions; and has received research support from Takeda. G.C., R.P., N.L., A.T., C.N., and C.S. are employees and stockholders of Pfizer Inc.

Supported by: Pfizer Inc.

Address correspondence to: Kevin L. Winthrop, MD, MPH, CEI/OHSU, 3375 SW Terwilliger Blvd, Portland, OR 97239 (winthrop@ohsu.edu).

(C) 2018 Crohn's \& Colitis Foundation. Published by Oxford University Press on behalf of Crohn's \& Colitis Foundation. This is an Open Access article distributed under the terms of the Creative Commons Attribution Non-Commercial License (http://creativecommons.org/ licenses/by-nc/4.0/), which permits non-commercial re-use, distribution, and reproduction in any medium, provided the original work is properly cited. For commercial re-use, please contact journals.permissions@oup.com

doi: $10.1093 /$ ibd/izy 131 Published online 30 May 2018 
Tofacitinib is an oral, small molecule Janus kinase (JAK) inhibitor that is being investigated for ulcerative colitis (UC). Tofacitinib preferentially inhibits cytokine receptors associated with JAK1 and/or JAK3 over cytokine receptors associated with JAK2 or JAK2/tyrosine kinase 2.8, As a result, the immune response is modulated via the downregulation of multiple cytokines shown to be important in the pathogenesis of UC (such as type 1 interferon, and interleukin [IL]-4, IL-5, IL-6, IL-9, IL-13, and IL-15). ${ }^{8,10-12}$ An increased risk of HZ has been noted with the use of tofacitinib and other JAK inhibitors in RA, and more recently with tofacitinib in psoriasis (PSO) and psoriatic arthritis. ${ }^{13-16}$ In addition to known risk factors for $\mathrm{HZ}$ (eg, age, and prednisone use), evaluation of tofacitinib development program data in RA has identified Asian race (highest risk in certain subregions such as Japan and Korea), tofacitinib dose, and concomitant steroid therapy as important risk factors for HZ. ${ }^{13,17}$ Accordingly, we undertook a retrospective evaluation of $\mathrm{HZ}$ cases, as reported within the tofacitinib clinical development program for UC, with the objectives of describing the outcomes of these cases, describing the overall risk of $\mathrm{HZ}$ in this setting, and identifying $\mathrm{HZ}$ risk factors among patients with UC using tofacitinib.

\section{METHODS}

\section{Ethical Considerations and Development Program Conduct}

The tofacitinib clinical development program for $\mathrm{UC}$ consisted of the following global studies (Fig. 1): (A) an 8-week phase II induction study (NCT00787202); (B) two 8-week phase III induction studies (OCTAVE Induction 1, NCT01465763, and OCTAVE Induction 2, NCT01458951) of identical design (with treatment up to week 9); (C) a 52-week phase III maintenance study (OCTAVE Sustain, NCT01458574) with treatment up to week 53; and (D) an ongoing, open-label, long-term extension (OLE) study (NCT01470612) with treatment up to 47 months (3.9 years) as of December 16, 2016. Tofacitinib $10 \mathrm{mg}$ twice daily (BID) was evaluated in the placebo-controlled induction studies. Tofacitinib 5 and $10 \mathrm{mg}$ BID were evaluated in the placebo-controlled maintenance study and the OLE study. Patients meeting clinical response criteria (decrease from baseline in total Mayo score of $\geq 3$ points and $\geq 30 \%$, with an accompanying decrease in rectal bleeding subscore $\geq 1$ or absolute rectal bleeding subscore $\leq 1$ ) at the end of the 8-week induction studies were re-randomized to receive placebo, tofacitinib 5-mg BID, or tofacitinib 10-mg BID doses during the 52-week maintenance study. Nonresponders from the induction studies were eligible for inclusion within the OLE study, as were those completing or withdrawing early, due to treatment failure, from the maintenance study. ${ }^{18}$ Concomitant oral corticosteroids were permitted during the phase II and III induction studies (maximum doses of 30 and $25 \mathrm{mg} / \mathrm{d}$, respectively), followed by mandatory taper to 0 after induction. Concomitant TNFi and immunosuppressants were prohibited.

All enrolled patients provided informed consent, and all participating institutions provided institutional review board approval before participation. The studies were conducted in compliance with the Declaration of Helsinki, the Good

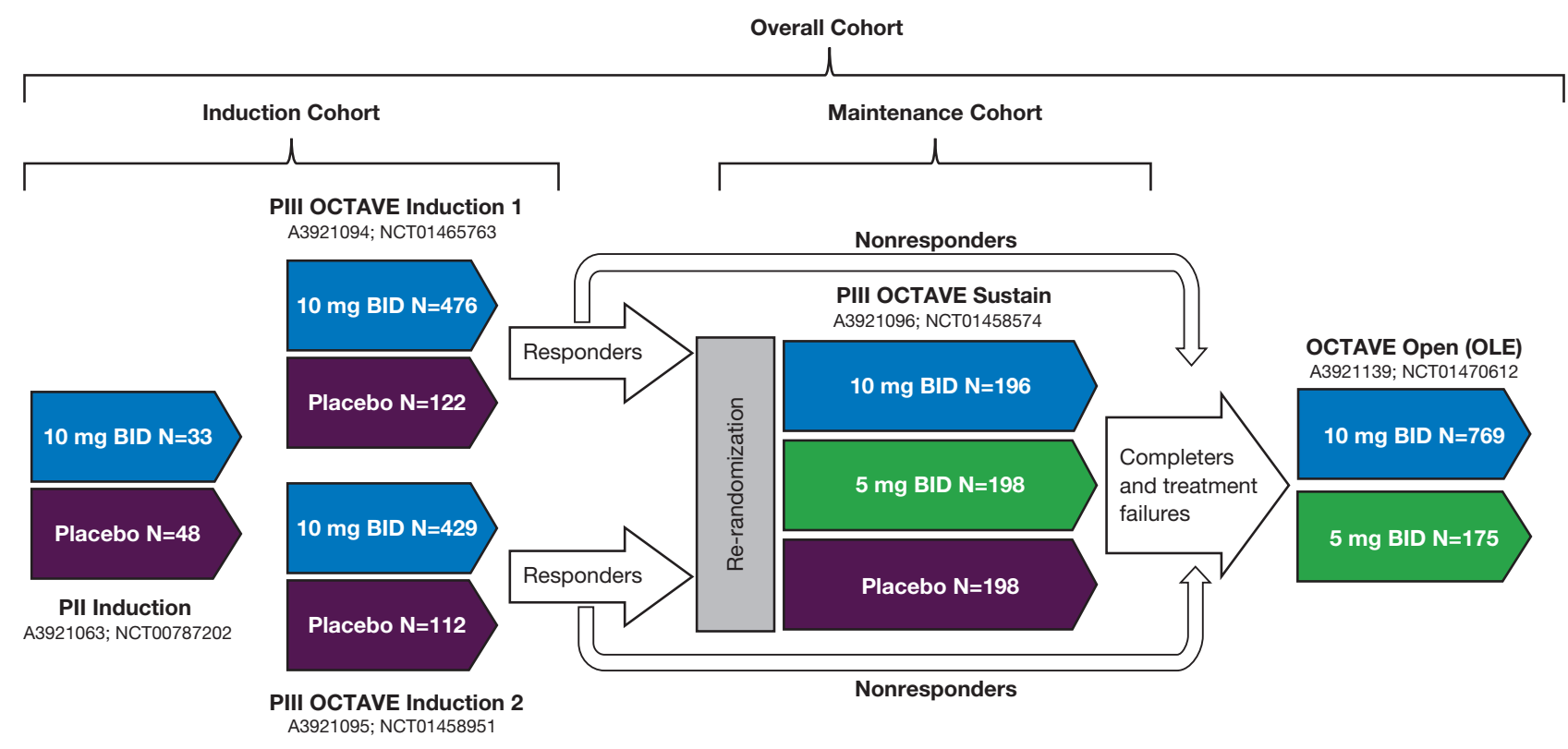

FIGURE 1. Overview of the tofacitinib development program for moderate to severe UC and the cohorts investigated. Patients meeting positive response criteria at the end of the 8-week OCTAVE Induction 1 and 2 studies were re-randomized to receive either tofacitinib 5-mg BID or tofacitinib 10-mg BID doses during the 52-week maintenance (OCTAVE Sustain) study. Nonresponders from the induction studies were eligible for inclusion within the OLE study, as were those completing the maintenance study. Abbreviations: $N$, number of patients treated; P, Phase. 
Clinical Practice guidelines of the International Conference on Harmonization, and relevant local country regulations.

\section{Analysis Cohorts}

For evaluation of $\mathrm{HZ}$ events, we constructed 3 evaluation cohorts (Fig. 1). The Induction Cohort included all patients entering the three 8-week induction studies (phase II and OCTAVE Induction 1 and 2 studies) and included all exposure time from weeks $0-9$. The Maintenance Cohort included all patients entering the 52-week phase III maintenance study (OCTAVE Sustain) and was restricted to their time within the study (weeks 0-53). The Overall Cohort included all patients treated with tofacitinib 5 or $10 \mathrm{mg}$ BID within all phase II, phase III, and ongoing OLE (OCTAVE Open) studies.

\section{HZ Case Finding and Adjudication}

For all studies, we searched all Medical Dictionary for Regulatory Activities terms reported by investigators containing text consistent with potential $\mathrm{HZ}$, using an interim data cutoff date of December 16, 2016. These terms included: "herpes zoster," "herpes zoster disseminated," "herpes zoster cutaneous disseminated," "ophthalmic herpes zoster," "herpes zoster oticus," "genital herpes zoster," "herpes zoster pharyngitis," "herpes zoster necrotizing retinopathy," "herpes zoster infection neurological," "herpes zoster meningitis," "herpes zoster meningomyelitis," and "herpes zoster meningoencephalitis." For all reported HZ, we collected descriptive clinical information for each patient. HZ events were graded as serious infection events if they met serious adverse event (AE) criteria. Cases reported as serious AEs, or those reported to involve $>1$ dermatome, were sent to an independent, blinded, external adjudication committee. Cases were adjudicated and considered "multidermatomal" if they involved 2 nonadjacent or 3-6 adjacent dermatomes, or "disseminated" if they involved $\geq 7$ dermatomes or visceral disease. Patients who experienced HZ serious infections were required to be discontinued.

\section{HZ IR Calculations and Evaluation of Comparative Risk by Dose}

We calculated the proportion of patients with $\mathrm{HZ}$ for the Induction Cohort. HZ incidence rates (IRs; patients with events per 100 patient-years [PY]; $95 \%$ confidence interval [CI]), stratified by race, age, history of diabetes mellitus at baseline, and other factors, were calculated for the Maintenance and Overall Cohorts. For all IR calculations, patients were censored at time of first event, study withdrawal or death, or end of follow-up (28 days after the end of the study), whichever came first. To examine whether HZ risk was modified by tofacitinib dose, we used data from the Maintenance Cohort, where patients received a fixed dose of tofacitinib 5 or 10-mg BID for 52 weeks. Because patients could modify their dose during the OLE portion of the study, we categorized patients as predominantly taking either a 5- or 10-mg BID dose, based on their average daily dose of tofacitinib during their entire participation in the program. Those patients with an average daily dose $\geq 15 \mathrm{mg}$ were categorized as $10 \mathrm{mg}$ BID for analysis purposes, and those below this threshold were considered 5-mg BID users. We also calculated IRs within the Overall Cohort, and by time windows of exposure, to evaluate whether rates changed over time (eg, exposure for $0-6$ months, $>6-12$ months, $>12-18$ months, etc.)

\section{Risk Factor Analysis}

We used prior studies in $\mathrm{RA}$ and $\mathrm{PsO}$ to guide initial exploration of potential $\mathrm{HZ}$ risk factors in univariate analysis. ${ }^{13,14}$ We used data from the Overall (phase II/phase III/ OLE) Cohort for this analysis and constructed Cox proportional regression models to explore the association between $\mathrm{HZ}$ and the following covariates: baseline corticosteroid dosing group (none, $>0$ to $<15 \mathrm{mg} / \mathrm{d}$ prednisone equivalent, $\geq 15 \mathrm{mg} / \mathrm{d}$, or unknown), age ( $<65, \geq 65$ years), age ( $<30,30$ to $<40,40$ to $<50, \geq 50$ years), age (continuous), race (white, black, Asian, other), race (Asian, other), race (white, other), geographic regions (North America, Europe, rest of the world), geographic regions (Japan/Korea/Taiwan, rest of the world), geographic regions (Japan/Korea, rest of the world), body weight ( $<90, \geq 90 \mathrm{~kg}$ ), body mass index (BMI; $<25,25$ to $<30$, $\geq 30$ ), BMI continuous, sex (F, M), diabetes mellitus ( $\mathrm{Y}, \mathrm{N})$, UC duration (by median $<6.3, \geq 6.3$ years), prior TNFi use ( $\mathrm{Y}, \mathrm{N}$ ), prior TNFi failure (Y,N), prior immunosuppressant use (Y, $\mathrm{N}$ ), tofacitinib dose group (predominant dose), UC disease severity by baseline total Mayo score $(<9, \geq 9)$, baseline total Mayo score (continuous), oral corticosteroid use at baseline (Y, $\mathrm{N}$ ), smoking status (current, never, ex-smoker; for malignancies), baseline absolute lymphocyte count $\left(<1.0, \geq 1.0 \times 10^{9} / \mathrm{L}\right)$, and absolute neutrophil count $\left(<1.5, \geq 1.5 \times 10^{9} / \mathrm{L}\right.$; continuous). Variables with a $P$ value $<0.10$ were entered into a stepwise model, and those variables from the stepwise model with $P<0.05$ were retained in the final model. Given that prior analyses have identified Asian race as a risk factor (Asian vs nonAsian), we retained this variable in the final model. ${ }^{13,14}$

\section{RESULTS}

Demographic and baseline characteristics are described in Table 1. A total of 1157 patients were treated with tofacitinib (1612.8 PY of exposure) within the clinical development program for UC. Within the 8-week induction studies (Induction Cohort), $1(0.4 \%)$ placebo-treated patient and 6 $(0.6 \%)$ tofacitinib-treated patients developed HZ. During the 52-week maintenance study (Maintenance Cohort), $1(0.5 \%)$ placebo-treated patient (who had finished tofacitinib $10 \mathrm{mg}$ BID within the Induction study 116 days earlier), $3(1.5 \%)$ 5-mg BID tofacitinib-treated patients, and $10(5.1 \%) 10-\mathrm{mg}$ BID tofacitinib-treated patients developed HZ (Table 2). Among all tofacitinib-treated patients in the Overall Cohort, we identified a total of $65(5.6 \%)$ patients who developed HZ during tofacitinib use, with an IR (95\% CI) of 4.07 (3.14-5.19). 


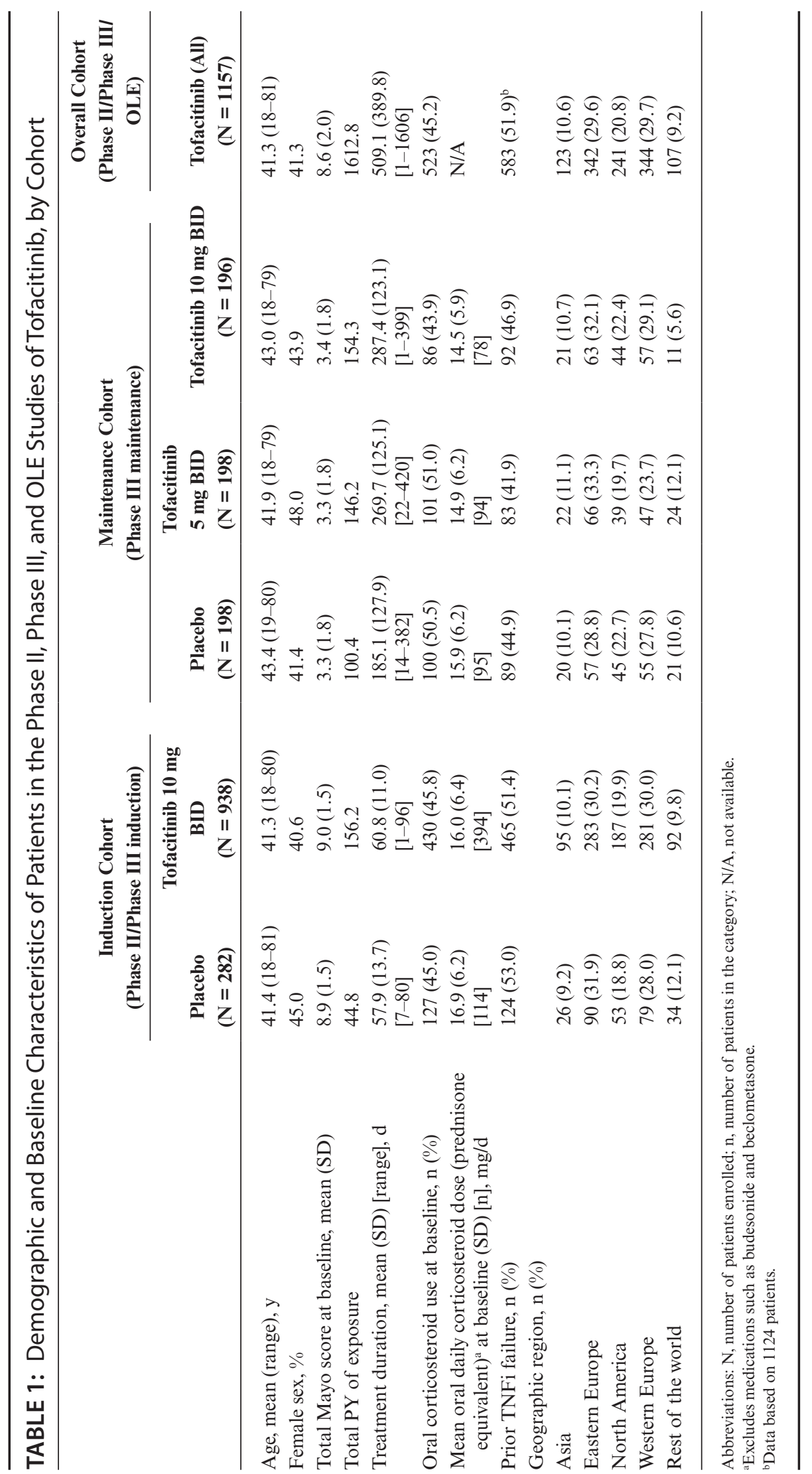


TABLE 2: HZ IRs ${ }^{\mathrm{a}}$ by Dose Within the Maintenance (Phase III Maintenance) and Overall (Phase II/Phase III/OLE) Cohorts

\begin{tabular}{|c|c|c|c|c|c|}
\hline & \multicolumn{3}{|c|}{ Maintenance Cohort (Phase III maintenance) } & \multicolumn{2}{|c|}{ Overall Cohort (Phase II/Phase III/OLE) } \\
\hline & $\begin{array}{c}\text { Placebo } \\
(\mathrm{N}=198)\end{array}$ & $\begin{array}{c}\text { Tofacitinib } 5 \mathrm{mg} \text { BID } \\
\qquad(\mathrm{N}=198)\end{array}$ & $\begin{array}{l}\text { Tofacitinib } 10 \mathrm{mg} \text { BID } \\
\qquad(\mathrm{N}=196)\end{array}$ & $\begin{array}{c}\text { Tofacitinib } 5 \mathrm{mg} \text { BID }^{\mathrm{a}} \\
(\mathrm{N}=186)\end{array}$ & $\begin{array}{c}\text { Tofacitinib } 10 \mathrm{mg} \mathrm{BID} \\
\qquad(\mathrm{N}=971)\end{array}$ \\
\hline $\begin{array}{l}\text { Patients with events, } \\
\text { n (\%) }\end{array}$ & $1(0.5)$ & $3(1.5)$ & $10(5.1)$ & $12(6.5)$ & $53(5.5)$ \\
\hline IR $(95 \% \mathrm{CI})$ & $0.97(0.02-5.42)$ & $2.05(0.42-6.00)$ & $6.64(3.19-12.22)$ & $3.45(1.78-6.02)$ & $4.25(3.18-5.56)$ \\
\hline
\end{tabular}

Only events occurring within 28 days after the last dose are included in this table for calculation of proportion and IR.

Abbreviations: $\mathrm{N}$, number of patients randomized and treated; n, number of patients in the category.

atients with events per $100 \mathrm{PY}$.

${ }^{b}$ Patients predominantly taking either a 5- or 10-mg BID dose.

Of the patients developing $\mathrm{HZ}, 28(43 \%)$ were female; the median age at the time of $\mathrm{HZ}$ onset (range) was 52 (18-80) years, and the median time to develop HZ (range) was 324 (13-1185) days. Although there were no recurrent HZ events, 4 of the 69 events were reported in patients who had already developed $\mathrm{HZ}$ within the study time frame. The majority of HZ events $(n=51,74 \%)$ involved 1 or 2 adjacent dermatomes. The remaining 18 events (in 17 patients) were judged to be multidermatomal (12 events in 11 patients) or disseminated (6 events in 6 patients). Among the disseminated events, 3 were diffuse cutaneous rashes, 2 involved ocular and skin disease, and 1 was an invasive case of $\mathrm{HZ}$ encephalitis. This patient was treated with intravenous acyclovir and oral valacyclovir and recovered. There were 4 events meeting serious AE criteria because of hospitalization, and there were no deaths resulting from HZ. Five of the $65(7.7 \%)$ patients with $\mathrm{HZ}$ discontinued the study, and $16(24.6 \%)$ patients with $\mathrm{HZ}$ had their tofacitinib temporarily withheld. Among the $69 \mathrm{HZ}$ events (in 65 patients), 58 (84.1\%) were treated with antiviral therapy and $3(4.6 \%)$ reported developing postherpetic neuralgia.

\section{Comparative Risk by Dose and Exposure Group}

Within the Maintenance Cohort, where tofacitinib dosing was fixed, a dose response was observed, with higher IRs observed among patients using tofacitinib $10 \mathrm{mg}$ BID compared with those dosed at tofacitinib $5 \mathrm{mg}$ BID (Table 2). In the Overall Cohort, IRs were higher in Asian patients, those with diabetes, those taking corticosteroids at baseline, those

TABLE 3: Stratified IRs $s^{a}$ for HZ Within the Overall Cohort

\begin{tabular}{|c|c|c|c|c|}
\hline & Subgroup & $\mathbf{N}$ & n $(\%)$ & IR $(95 \% \mathrm{CI})$ \\
\hline \multirow[t]{2}{*}{ Age } & $<65 \mathrm{y}$ & 1080 & $54(5.0)$ & $3.65(2.74-4.76)$ \\
\hline & $\geq 65 \mathrm{y}$ & 77 & $11(14.3)$ & $9.55(4.77-17.08)$ \\
\hline & No & 1109 & $59(5.3)$ & $3.88(2.95-5.00)$ \\
\hline Oral corticosteroid use at baseline & Yes & 523 & $34(6.5)$ & $5.14(3.56-7.18)$ \\
\hline & No & 541 & $23(4.3)$ & $2.78(1.77-4.18)$ \\
\hline \multirow[t]{2}{*}{ Prior immunosuppressant treatment } & Yes & 838 & $50(6.0)$ & $4.18(3.10-5.51)$ \\
\hline & No & 286 & $14(4.9)$ & $3.57(1.95-5.99)$ \\
\hline \multirow[t]{2}{*}{ Race } & White & 927 & $44(4.7)$ & $3.50(2.54-4.70)$ \\
\hline & Black & 10 & $0(0.0)$ & $0.00(0.00-23.30)$ \\
\hline
\end{tabular}

Only events occurring within 28 days after the last dose are included in this table for calculation of proportion and IR.

Abbreviations: $\mathrm{N}$, number of patients randomized and treated; $n$, number of patients with events.

aPatients with events per $100 \mathrm{PY}$. 


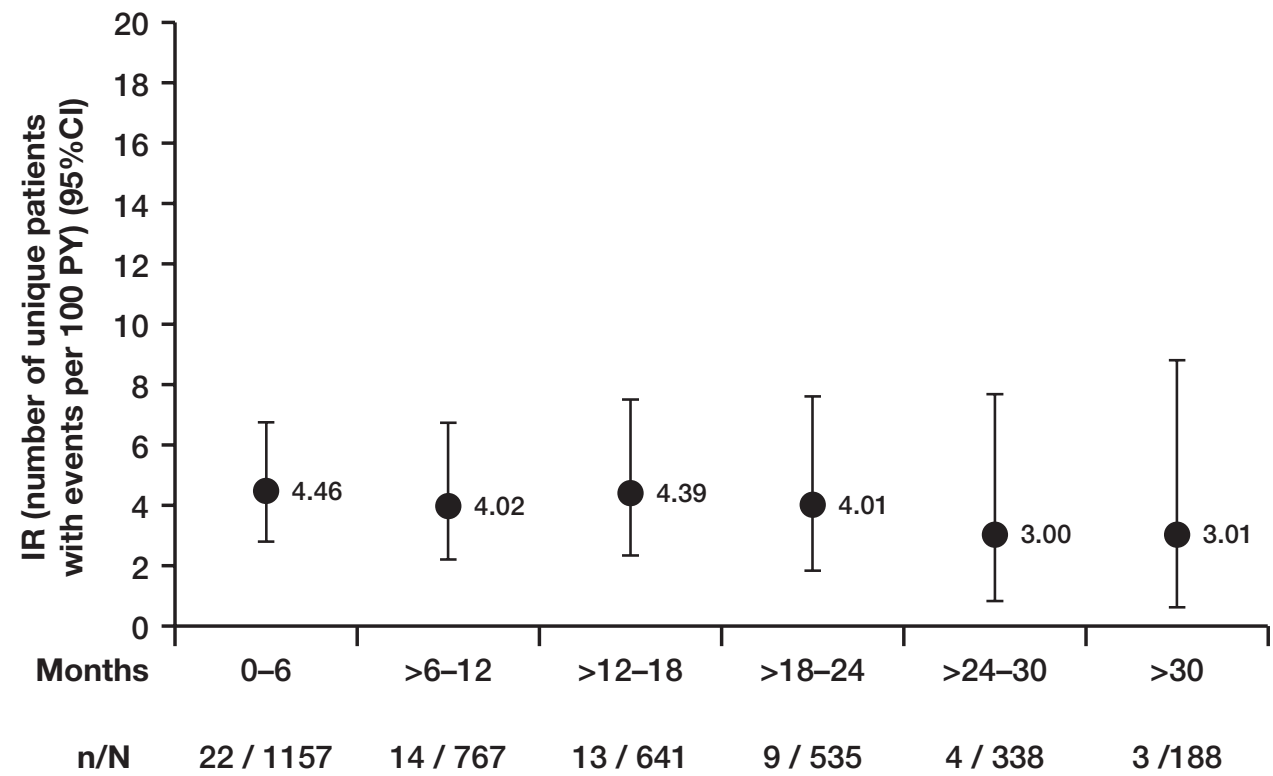

FIGURE 2. IRs of $\mathrm{HZ}$ by time interval in Overall Cohort (phase II/phase III/OLE). Abbreviations: $\mathrm{N}$, number of evaluable patients; $n$, number of unique patients with $\mathrm{HZ}$ infection.

with prior TNFi failure, and those age $\geq 65$ years (Table 3). In the Overall Cohort, analyses of IR by time interval showed that $\mathrm{HZ}$ risk did not increase with longer treatment duration (Fig. 2).

\section{Cox Proportional Hazards Model Evaluation of HZ Risk Factors}

In univariate analysis, several variables were associated with HZ $(P \leq 0.1)$. These included Asian race, smoking status, advancing age, history of prior TNFi use, history of prior TNFi failure, white race, region, BMI, prednisone dose, corticosteroid use at baseline, and diabetes. These were included in the final multivariate stepwise model, which identified age (for each 10-year increment; hazard ratio [HR], 1.58; 95\% CI, $1.34-1.87 ; P<0.0001)$ and prior TNFi failure (HR, 1.92; $95 \%$ CI, $1.15-3.21 ; P=0.0122$ ) as significant independent risk factors for infection. Asian patients had a higher risk of HZ, although this was not statistically significant within the multivariate model (HR, 1.76; 95\% CI, 0.97-3.19; $P=0.0612$ ) (Fig. 3).

\section{DISCUSSION}

We have conducted the first analysis examining the risk of $\mathrm{HZ}$ in patients with UC using tofacitinib. Similar to our previous analysis of $\mathrm{HZ}$ within the tofacitinib development program for RA, ${ }^{13}$ we observed higher incidence rates of $\mathrm{HZ}$

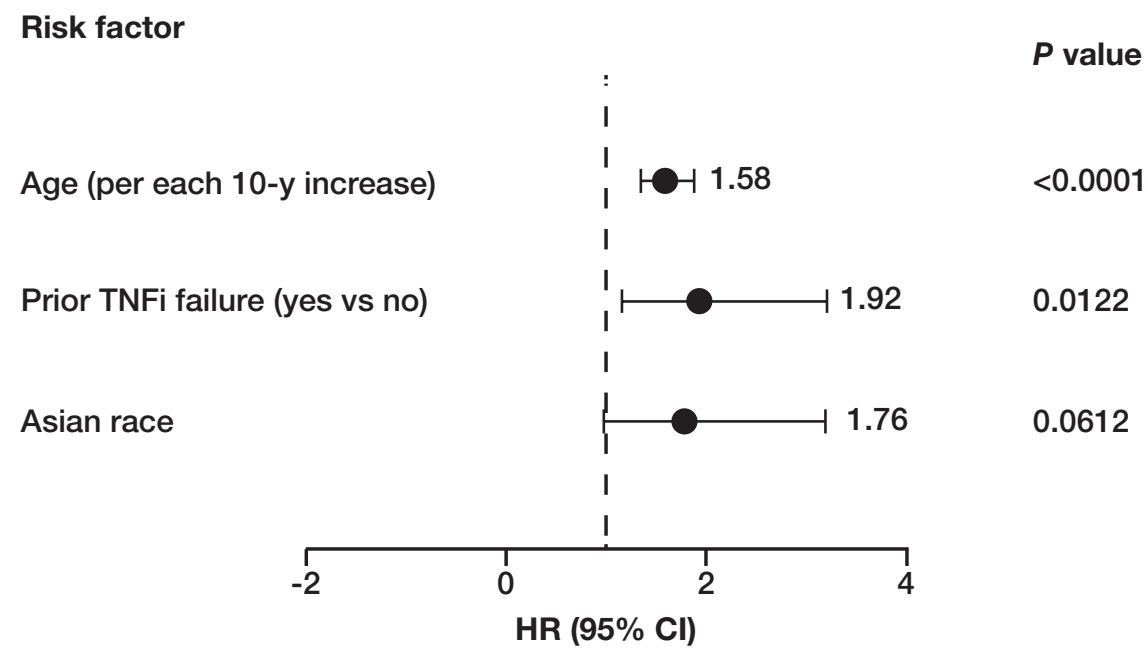

FIGURE 3. Cox proportional hazards model of risk factors predicting $\mathrm{HZ}$ among UC patients using tofacitinib. 
among older individuals, Asians, those with prior TNFi use, those using 10-mg BID doses of tofacitinib, and those with corticosteroid use at baseline. ${ }^{13}$ Multivariate modeling identified age and prior TNFi failure as independent risk factors for $\mathrm{HZ}$ among UC patients using tofacitinib.

The increased risk of $\mathrm{HZ}$ with tofacitinib seems to represent a class effect, with increased risks reported with other approved and in-development JAK inhibitors. ${ }^{19}$ Further, the reported rates in the tofacitinib and baricitinib development programs for RA have been driven in part by a 1.5 - to 2 -fold increase in risk within some Asian countries compared with North American or European regions. ${ }^{15,20}$ The reason for the higher risk in Asia under conditions of JAK blockade is unclear. One genetic analysis identified 2 single nucleotide polymorphisms (SNPs) that conferred increased risk within Japanese and Korean populations. However, these SNPs were infrequently present and explained only a small percentage of the increased incidence noted within the tofacitinib development program for RA. ${ }^{8}{ }^{20}$ Interestingly, although there is increased risk for $\mathrm{HZ}$ with tofacitinib, manifestations of this disease are largely limited to cutaneous presentations. ${ }^{13,}{ }^{14}$ Although 1 case of visceral disease (encephalitis) occurred within the UC development program, nearly all prior reported cases with tofacitinib, and other JAK inhibitors, ${ }^{15,}, 19$ have involved monodermatomal or multidermatomal skin or ocular disease only. The mechanism by which tofacitinib or other JAK inhibitors increase the risk of $\mathrm{HZ}$ is unknown, although it could be related to a variety of potential JAK/signal transducer and activator of transcription (STAT)-related mechanisms. Cell-mediated immunity to VZV can be measured in vitro by VZV-stimulated CD4 lymphocyte interferon-gamma responses, and decreased responses are correlated with an increased risk of HZ. ${ }^{21-23}$ Although the effect of tofacitinib on such an assay has not been directly measured, tofacitinib has been shown to modestly decrease lymphocyte counts and CD4 cell proliferation. ${ }^{24}$ Other cells important in viral immunity include natural killer (NK) cells, the levels of which decline during tofacitinib use, ${ }^{25}$ and it is possible that there is diminishment of NK cell function. The latter was suggested in vitro using a lymphoproliferative cell line exposed to tofacitinib but has never been tested directly with $\mathrm{VZV} .^{26}$ Lastly, it is likely that inhibition of type 1 and 2 interferons via tofacitinib, baricitinib, and other JAK inhibitors is responsible for interfering with the innate antiviral immunity associated with VZV. Type 1 and type 2 interferon signaling rely upon the JAK1 receptor, which is inhibited in part by all of these compounds. ${ }^{27}$ Further, data from studies using anti-interferon alpha monoclonal antibodies for systemic lupus erythematosus also suggest a high rate of $\mathrm{HZ},{ }^{28}$ and further highlight the importance of interferon signaling in controlling $\mathrm{HZ}$ reactivation and clinical disease. ${ }^{6,7}$

In terms of evaluating risk factors, our modeling is consistent with prior tofacitinib analyses in finding that $\mathrm{HZ}$ is more likely to occur in older individuals, those in Asia, and those with prior biologic use. ${ }^{13,14}$ Although concomitant baseline steroid use was not an independent risk factor in our analysis, IRs in our study were higher for those using steroids, a well-known dose-dependent risk factor for HZ. ${ }^{13,29}$ Within RA, patients using tofacitinib in the absence of steroids have been shown to be at lower risk than those using steroids. ${ }^{20}$ Interestingly, the IRs observed within the UC program were similar to those observed in $\mathrm{RA},{ }^{20}$ despite the age distribution of the UC program being younger and more similar to that of the PsO program, where lower IRs were seen. ${ }^{13}$ This may reflect a difference in concomitant prednisone use (ie, more common in $\mathrm{UC}$ than $\mathrm{PsO}$, and higher doses used in UC vs RA) and potential differences in underlying disease.

Vaccination remains an important tool for $\mathrm{HZ}$ risk management and is likely to benefit all patients with immunemediated diseases who receive treatment, particularly those using JAK inhibitors. Prior analyses within RA patients using the live zoster vaccine before starting tofacitinib suggest that patients tend to develop appropriate vaccine-induced immune responses, which are only mildly reduced compared with the general population. ${ }^{30,31}$ Although the long-term effectiveness of this vaccine remains unknown in the setting of autoimmune disease, population-based data suggest efficacy similar to the general population, and it is recommended for use before any biologic or JAK inhibitor in those over 50 years of age. ${ }^{32}$ Because this vaccine is live, it is contraindicated in those actively using biologics, JAK inhibitors, or high-dose steroids (doses $\geq 20 \mathrm{mg}$ for $>2$ weeks). ${ }^{1}$ This contraindication complicates its use, as patients must be off these therapies while receiving the vaccine. Currently there is 1 ongoing, large randomized controlled trial evaluating the safety and immunogenicity of this vaccine among those patients age $\geq 50$ years actively using TNFi, including patients with UC (VERVE trial, NCT02538341). Lastly, a new, adjuvanted, nonlive subunit vaccine has just been approved in the United $\mathrm{States}^{33}$ and Canada ${ }^{34}$ for prevention of $\mathrm{HZ}$ in adults age 50 years and older, with reported high efficacy rates, but also high rates of reactogenicity. ${ }^{35}$ Whether this vaccine will cause flares of underlying autoimmune disease is unknown. Study of this vaccine in patients with autoimmune diseases, including younger patients age $<50$ years, to determine whether the vaccine is as efficacious as the live zoster vaccine and whether it induces flares should be undertaken.

In summary, we have evaluated the risk of $\mathrm{HZ}$ among a cohort of UC patients using tofacitinib. As with prior analyses of tofacitinib in $\mathrm{RA}$ and $\mathrm{PsO}$, higher rates of $\mathrm{HZ}$ were observed among Asian patients, older individuals, and those using higher doses of tofacitinib. Additionally, similarly to these prior programs, the majority of cases were nonserious, cutaneous, and limited in their distribution, although the 1 visceral case highlights the potential for more serious manifestations of $\mathrm{HZ}$ in this setting. When HZ occurs, antiviral therapy should be considered and tofacitinib temporarily stopped until resolution. Efforts should be made to evaluate the effectiveness of $\mathrm{HZ}$ 
vaccination in preventing $\mathrm{HZ}$ among the high-risk populations identified within our study.

\section{ACKNOWLEDGMENTS}

Medical writing support under the guidance of the authors was provided by Sandrine M. Dupré, PhD, at CMC Connect, a division of Complete Medical Communications Ltd, Manchester, UK and was funded by Pfizer Inc, New York, NY, USA in accordance with Good Publication Practice (GPP3) guidelines (Ann Intern Med. 2015;163:461-464).

\section{REFERENCES}

1. Harpaz R, Ortega-Sanchez IR, Seward JF; Advisory Committee on Immunization Practices (ACIP) Centers for Disease Control and Prevention (CDC). Prevention of herpes zoster: recommendations of the Advisory Committee on Immunization Practices (ACIP). MMWR Recomm Rep. 2008;57:1-30; quiz CE2.

2. Schmader KE. Epidemiology and impact on quality of life of postherpetic neuralgia and painful diabetic neuropathy. Clin J Pain. 2002;18:350-354.

3. Schmader KE, Sloane R, Pieper C, et al. The impact of acute herpes zoster pain and discomfort on functional status and quality of life in older adults. Clin J Pain. 2007; $23: 490-496$

4. Smitten AL, Choi HK, Hochberg MC, et al. The risk of herpes zoster in patients with rheumatoid arthritis in the United States and the United Kingdom. Arthritis Rheum. 2007;57:1431-1438.

5. Yun H, Yang S, Chen L, et al. Risk of herpes zoster in autoimmune and inflammatory diseases: implications for vaccination. Arthritis Rheumatol. 2016;68:2328-2337.

6. Gupta G, Lautenbach E, Lewis JD. Incidence and risk factors for herpes zoster among patients with inflammatory bowel disease. Clin Gastroenterol Hepatol. 2006;4:1483-1490.

7. Long MD, Martin C, Sandler RS, et al. Increased risk of herpes zoster among 108604 patients with inflammatory bowel disease. Aliment Pharmacol Ther. 2013;37:420-429.

8. Clark JD, Flanagan ME, Telliez JB. Discovery and development of Janus kinase (JAK) inhibitors for inflammatory diseases. J Med Chem. 2014;57:5023-5038.

9. Meyer DM, Jesson MI, Li X, et al. Anti-inflammatory activity and neutrophil reductions mediated by the JAK1/JAK 3 inhibitor, CP-690,550, in rat adjuvant-induced arthritis. $J$ Inflamm (Lond). 2010;7:41.

10. Ungaro R, Mehandru S, Allen PB, et al. Ulcerative colitis. Lancet. 2017;389:1756-1770.

11. Neurath MF. Cytokines in inflammatory bowel disease. Nat Rev Immunol. 2014; 14:329-342.

12. Izzo R, Bevivino G, Monteleone G. Tofacitinib for the treatment of ulcerative colitis. Expert Opin Investig Drugs. 2016;25:991-997.

13. Winthrop KL, Yamanaka H, Valdez H, et al. Herpes zoster and tofacitinib therapy in patients with rheumatoid arthritis. Arthritis Rheumatol. 2014;66:2675-2684.

14. Winthrop KL, Lebwohl M, Cohen AD, et al. Herpes zoster in psoriasis patients treated with tofacitinib. J Am Acad Dermatol. 2017;77:302-309.

15. Winthrop KL, Lindsey S, Weinblatt M, et al. Herpes zoster in patients with moderate to severe rheumatoid arthritis treated with baricitinib. Arthritis Rheumatol. 2016;68:Abstract 3027
16. Burmester G, Fitzgerald $\mathrm{O}$, Winthrop $\mathrm{K}$, et al. Integrated safety summary of tofacitinib in psoriatic arthritis clinical studies. Arthritis Rheumatol. 2017;69:Abstract 616.

17. Winthrop KL, Curtis JR, Lindsey S, et al. Herpes zoster and tofacitinib: the risk of concomitant nonbiologic therapy. Arthritis Rheumatol. 2015;67:Abstract 559.

18. Sandborn WJ, Su C, Sands BE, et al; OCTAVE Induction 1, OCTAVE Induction 2 , and OCTAVE Sustain Investigators. Tofacitinib as induction and maintenance therapy for ulcerative colitis. $N$ Engl J Med. 2017;376:1723-1736.

19. Winthrop KL. The emerging safety profile of JAK inhibitors in rheumatic disease. Nat Rev Rheumatol. 2017;13:234-243.

20. Winthrop KL, Curtis JR, Lindsey S, et al. Herpes zoster and tofacitinib: clinical out comes and the risk of concomitant therapy. Arthritis Rheumatol. 2017;69:1960-1968.

21. Berger R, Florent G, Just M. Decrease of the lymphoproliferative response to varicella-zoster virus antigen in the aged. Infect Immun. 1981;32:24-27.

22. Levin MJ, Smith JG, Kaufhold RM, et al. Decline in varicella-zoster virus (VZV)-specific cell-mediated immunity with increasing age and boosting with a high-dose VZV vaccine. $J$ Infect Dis. 2003;188:1336-1344.

23. Sadaoka K, Okamoto S, Gomi Y, et al. Measurement of varicella-zoster virus (VZV)-specific cell-mediated immunity: comparison between VZV skin test and interferon-gamma enzyme-linked immunospot assay. J Infect Dis. 2008;198:1327-1333.

24. Maeshima K, Yamaoka K, Kubo S, et al. The JAK inhibitor tofacitinib regulates synovitis through inhibition of interferon- $\gamma$ and interleukin-17 production by human CD4+ T cells. Arthritis Rheum. 2012;64:1790-1798.

25. Cohen SB, Tanaka Y, Mariette X, et al. Long-term safety of tofacitinib for the treatment of rheumatoid arthritis up to 8.5 years: integrated analysis of data from the global clinical trials. Ann Rheum Dis. 2017;76:1253-1262.

26. Nocturne G, Tahmasebi F, Boudaoud S, et al. Tofacitinib is associated with an impaired function of NK cells and a defective immunosurveillance against B-cell lymphomas. Arthritis Rheumatol. 2016;68(Suppl 10):3514.

27. Malmgaard L. Induction and regulation of IFNs during viral infections. $J$ Interferon Cytokine Res. 2004;24:439-454.

28. Petri M, Wallace DJ, Spindler A, et al. Sifalimumab, a human anti-interferonmonoclonal antibody, in systemic lupus erythematosus: a phase I randomized, controlled, dose-escalation study. Arthritis Rheum. 2013;65:1011-1021.

29. Yun H, Xie F, Delzell E, et al. Risks of herpes zoster in patients with rheumatoid arthritis according to biologic disease-modifying therapy. Arthritis Care Res. 2015;67:731-736

30. Winthrop KL, Wouters AG, Choy EH, et al. The safety and immunogenicity of live zoster vaccination in patients with rheumatoid arthritis before starting tofacitinib: a randomized phase II trial. Arthritis Rheumatol. 2017;69:1969-1977.

31. Oxman MN, Levin MJ, Johnson GR, et al; Shingles Prevention Study Group A vaccine to prevent herpes zoster and postherpetic neuralgia in older adults. $N$ Engl J Med. 2005;352:2271-2284.

32. Zhang J, Xie F, Delzell E, et al. Association between vaccination for herpe zoster and risk of herpes zoster infection among older patients with selected immune-mediated diseases. JAMA. 2012;308:43-49.

33. US Food and Drug Administration. SHINGRIX (Zoster Vaccine Recombinant, Adjuvanted) suspension for intramuscular injection, highlights of prescribing information. 2017. https://www.fda.gov/downloads/BiologicsBloodVaccines/ Vaccines/ApprovedProducts/UCM581605.pdf. Accessed November 6, 2017.

34. Health Canada. SHINGRIX (Zoster Vaccine Recombinant, Adjuvanted) suspension for injection. 2017. http://ca.gsk.com/media/1350785/patient-information-english.pdf. Accessed November 6, 2017.

35. Lal H, Cunningham AL, Godeaux O, et al; ZOE-50 Study Group. Efficacy of an adjuvanted herpes zoster subunit vaccine in older adults. $N$ Engl $J$ Med. 2015;372:2087-2096. 